FOUNDATIONS OF COMPUTING AND DECISION SCIENCES

Vol. 39

No. 3

DOI: $10.2478 /$ fcds-2014-0010

ISSN 0867-6356

e-ISSN 2300-3405

\title{
AN EFFECTIVE METHOD TO EVALUATE THE SCIENTIFIC RESEARCH PROJECTS
}

\author{
Xiaohui $\mathrm{HOU}^{1}$, Lei HUANG${ }^{2}$, Xuefei $\mathrm{LI}^{3}$
}

\begin{abstract}
The evaluation of the scientific research projects is an important procedure before the scientific research projects are approved. The BP neural network and linear neural network are adopted to evaluate the scientific research projects in this paper. The evaluation index system with 12 indexes is set up. The basic principle of the neural network is analyzed and then the BP neural network and linear neural network models are constructed and the output error function of the neural networks is introduced. The Matlab software is applied to set the parameters and calculate the neural networks. By computing a real-world example, the evaluation results of the scientific research projects are obtained and the results of the BP neural network, linear neural network and linear regression forecasting are compared. The analysis shows that the BP neural network has higher efficiency than the linear neural network and linear regression forecasting in the evaluation of the scientific research projects problem. The method proposed in this paper is an effective method to evaluate the scientific research projects.
\end{abstract}

Keywords: effective method, evaluation, scientific research projects, index system, neural network.

\section{Introduction}

The evaluation of the scientific research projects (SRP) is an important procedure before the scientific research projects are set up. All of the science and technology management departments in different levels in China have paid much attention to the evaluation of the scientific research projects. Normally, in the scientific research project application process, there are more than one applicant and thus how to choose the best and most suitable research teams among the applicants, how to evaluate the scientific research project effectively become an important theoretical research topic. Many scholars research on the evaluation of scientific research projects in recent years, and they have applied a variety of

\footnotetext{
'Science and Technology Division, Beijing Jiaotong University, 100044 Beijing, China, Corresponding author e-mail: xhhou@bjtu.edu.cn

${ }^{2}$ School of Economics and Management, Beijing Jiaotong University, 100044 Beijing, China

3 School of Traffic and Transportation, Beijing Jiaotong University, 100044 Beijing, China
} 
methods and get a series of research findings. Liberatore (1987) [4] adopted an extension of the Analytic Hierarchy Process (AHP) to study on the industrial Research and Development (R\&D) project selection problem, and he used the spreadsheet model to rank the projects and then adopted the cost-benefit analysis and integer programming to support the resource allocation. Schmidt and Freeland (1992) [10] analyzed and reviewed the quantitative models which are used in $R \& D$ project selection, including the introduction of $R \& D$ project selection problem, classical decision models which focused on outcomes, systems models which focused on the insights of this problem, the structural, functional and quantitative models of R\&D systems and they also analyzed the future research needs in this field. Henriksen and Traynor (1999) [3] overviewed the most recent literature of R\&D project selection and proposed an improved and practical scoring tool to evaluate the R\&D projects which ranked the projects based on relevance, risk, reasonableness, and return criteria. They also designed a scoring algorithm which included tradeoffs between the criteria and calculated the relative measure of project value. Meade and Presley (2002) [7] analyzed the requirements of $R \& D$ project selection process and applied the analytic network process (ANP) method to evaluate the value of the $\mathrm{R} \& \mathrm{D}$ project proposals. They also proposed a generic ANP model which included the decision levels, actors, stages, categories, and individual metrics. And they used a case study to show the implementation of the proposed model. Mohanty et al. (2005) [8] used both fuzzy ANP and fuzzy cost analysis to make the decision of R\&D project selection. They also used the fuzzy set theory to overcome the vagueness in the preferences. In their study, they analyzed the different phases of R\&D projects, the different factors which affected the decision making, and enterprise functions. They also introduced the background of solution methodology and showed the methods to calculate the weights of different attributes and showed the interdependent components and cost analysis. Carlsson et al. (2005) [2] adopted the fuzzy real options approach to evaluate the R\&D projects. They analyzed the existing researches and then showed the real options for R\&D portfolios, and introduced the fuzzy approach to real option valuation, and discussed the implementation of the proposed method. Song and Wang (2011) [12] put forward an evaluation method of scientific research projects based on the weighted optimum order numbers method, the ranking example showed that this method is effective and accurate. Wang and Song (2008) [13] adopted the F-AHP method to evaluate the scientific research projects, they combined the fuzzy comprehensive evaluation method and analytic hierarchy process (AHP) together, determined the indexes weight by using the related software and then evaluate the fuzzy indexes by using fuzzy comprehensive evaluation and got the evaluation result. Lin (2006) [6] used the TOPSIS method to discuss the evaluation method of scientific research projects and applied the method in an example. Cao and Chai (2007) [1] adopted the fuzzy comprehensive evaluation to evaluate the scientific research projects. They analyzed the advantages of fuzzy comprehensive evaluation and analyzed the evaluation result by calculating an example, the result showed the fuzzy comprehensive evaluation is easy operation and could be trust. Lin (2005) [5] used the method of set pair analysis to combine the scientific research projects and indexes and found the relationship between them. And then they ordered the scientific research projects by their quality.

Overall, the above methods are traditional evaluation methods, and few use the advanced intelligent evaluation method. In order to implement the methods, the traditional evaluation methods usually need the experts/reviewers to score all evaluation indexes in all of the scientific research projects. Because there are often many indexes in the evaluation 
system and many projects to be evaluated, the tasks for the experts/reviewers are very heavy, and it maybe is not possible to score all of the indexes in all projects. Naturally, we will think about whether we could evaluate all of the projects only under evaluating part of them. For this purpose, we study on the feasibility and effectiveness of adopting the neural network method to evaluate the scientific research projects. The neural network method is an intelligent comprehensive evaluation method which has a strong ability of nonlinear fitting and could map arbitrarily complex nonlinear relationship. The neural network is able to simulate and reproduce the behaviors of the human decision-making and it is suitable to reproduce the index factors involved in the evaluation of the scientific research projects. It needs only part of the scientific research projects to train the neural network model and could evaluate the other projects by using it. Therefore, we adopt the neural network methods for the evaluation of the scientific research projects in this paper.

The remainder of this study is organized as follows: section 2 constructs the evaluation index system of the scientific research projects and section 3 introduces the basic principle of the neural networks and the research idea of this study, constructs the BP neural network model and linear neural network model, and shows the output error function of the proposed neural networks. Section 4 uses an example to analyze the performances of the proposed BP neural network and linear neural network. In the end, section 5 makes a conclusion of this study.

\section{Construction of Evaluation Index System}

The evaluation index system of the scientific research projects should strive to reflect the rationality, feasibility, innovation of the projects, and the ability of the applicants to complete the projects (Pan and Liu, 2004) [9], and reflect the basic situation of the project applications as objectively as possible. Based on the characteristics of the neural network models and under the ample investigation and in-depth analysis, the evaluation index system of the scientific research projects contains 12 indicators is set up, as shown in Table 1.

Table 1. Evaluation index system of the scientific research projects

\begin{tabular}{|c|c|c|c|}
\hline \multicolumn{4}{|c|}{ Evaluation index system of the scientific research projects } \\
\hline $\begin{array}{l}\text { Necessity of the topic } \\
\text { (NOT) }\end{array}$ & $\begin{array}{c}\text { Feasibility of the } \\
\text { research objectives } \\
\text { (FORO) }\end{array}$ & $\begin{array}{l}\text { Scientificity of the } \\
\text { technical solutions } \\
\text { (SOTS) }\end{array}$ & $\begin{array}{l}\text { Degree of the } \\
\text { technological } \\
\text { innovation } \\
\text { (DOTI) }\end{array}$ \\
\hline $\begin{array}{l}\text { Budget rationality } \\
\text { (BR) }\end{array}$ & $\begin{array}{l}\text { Rationality of the } \\
\text { expected results } \\
\text { (ROER) }\end{array}$ & $\begin{array}{c}\text { Research foundation } \\
\text { of the applicant } \\
\text { (RFOA) }\end{array}$ & $\begin{array}{l}\text { Human resources for } \\
\text { science and } \\
\text { technology } \\
\text { (HRST) }\end{array}$ \\
\hline $\begin{array}{l}\text { Experimental } \\
\text { conditions } \\
\text { (EC) }\end{array}$ & $\begin{array}{c}\text { Degree of technical } \\
\text { risk } \\
\text { (DOTR) }\end{array}$ & $\begin{array}{c}\text { Difficulty level of the } \\
\text { achievements } \\
\text { transformation } \\
\text { (DLOAT) }\end{array}$ & $\begin{array}{l}\text { Industrial prospects } \\
\text { (IP) }\end{array}$ \\
\hline
\end{tabular}


The indexes which are shown in Table 1 are explained as following: Necessity of the topic (NOT) reflects the necessity that whether we should choose the topics which the applicants propose. Feasibility of the research objectives (FORO) shows that whether the objectives in the applications are suitable or not, whether the objectives could be reached in the research period. Scientificity of the technical solutions (SOTS) means that whether the technical solutions in the research processes are scientific or not, and the high scientificity could ensure the scientific research projects could be studied smoothly and effectively. The purposes of the scientific research projects are to find the new theory and method to solve the existing problems, thus the degree of the technological innovation (DOTI) will be considered sufficiently in the evaluation of the scientific research projects. Budget rationality (BR) means that whether the budgets in the proposed scientific research projects are reasonable or not, and the government/school/company wants to use the money efficient and reduce the unnecessary spending. Rationality of the expected results (ROER) reflects that the research teams should propose the reasonable expected results of the scientific research projects, neither too big nor too small. The research foundation of the applicant (RFOA) is very important in the evaluation of the scientific research projects. If the applicants have abundant research foundations and research achievements, the research teams of the applicants have much more experiences than the teams which are lack of experiences in this field. And the applicants who have good research foundation will master the scientific research projects easier. Human resources for science and technology (HRST) reflect the human resources level of the applicants, whether the applicants have sufficient research people to complete the scientific research projects which they apply. Experimental conditions (EC) shows the equipment level of the applicants. The research teams which have better experimental conditions have the advantage of applying the scientific research projects and the choice probability of those teams are larger than the other teams. Not all scientific research projects could success and reach the research objectives in the end of the projects research. We have to face the technical risk that exists in the scientific research projects and the degree of technical risk (DOTR) is considered carefully in the evaluation of scientific research projects. The projects with lower risk will be paid more attention than the projects with higher risk. Difficulty level of the achievements transformation (DLOAT) shows that the scientific research projects are not only for researching, but also for transformation. The scientific research projects which are easier to transform in reality than the others have more advantages in the evaluation of the scientific research projects. Industrial prospects (IP) reflect the relationship between the scientific research inputs and outputs. If the scientific research projects have high industrial prospects, these scientific research projects will have priorities in this aspect.

\section{Basic Principle of the Neural Network and Construction of the Models}

\subsection{Basic Principle of the Neural Network}

Artificial neural network is a mathematical model that simulates the structure and function of the human brains and neural network behavioral characteristics, and process the 
information under distributed parallel processing. Artificial neural network has good adaptability of the nonlinear information, and could deal with such data better, and overcome the defects of the traditional methods. Artificial neural network has the ability to acquire knowledge by learning and solve the problems, analyze and process the data which enter into the system by "training" process, and grasp the potential rules and characteristics of the input data, and finally output the results according to the training process and complete the model construction.

Artificial neural network was first proposed in 1943, and there are more than 40 different types of neural network models after the development and evolution in recent 70 years, and these models can be applied to optimize, the pattern recognition and the prediction, and other fields. The different type of the neural network models has the different adaptabilities to the different input data and potential rules. At the same time, in order to avoid the uncertainty of the calculation results by using a single neural network model, and select the most suitable neural network model according to the characteristics of input data, we pick out the BP neural network and the linear neural network as the research models in the evaluation of the scientific research projects. By comparing the computed results of the two neural network models, we choose the best model as the standard model, and process and compute the evaluation data by using the standard model. In this paper, the construction idea of the neural network models to evaluate the scientific research projects is shown in Figure 1.

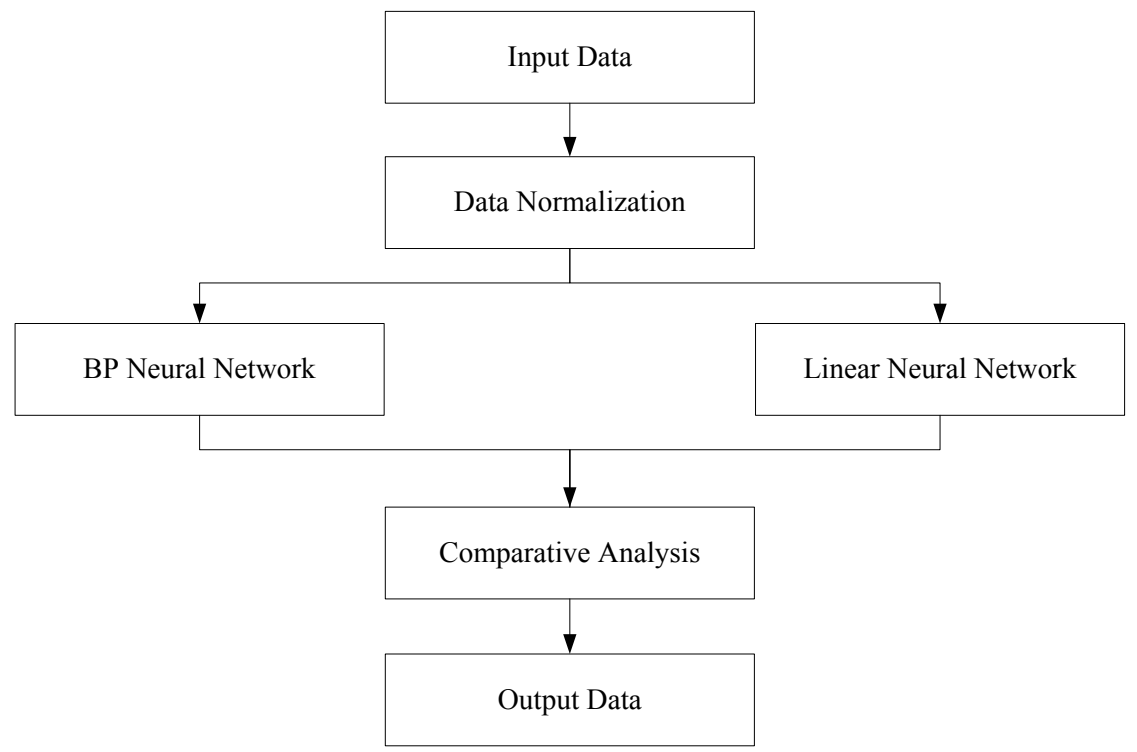

Figure 1. Construction idea of the neural network models

It should be observed that in the data normalization process in Figure 1, because of the scores of different indexes are obtained by the 100 scoring method, we have to normalize the scores between 0 and 1 . That is because that for the neural network models, if the values are between 0 and 1, it will speed up the computing process of the neural networks and we could get the computed results much more quickly. After the computing process, we have 
to convert the output values into the primary forms and then compare and analyze the output values of different neural networks.

\subsection{Construction of the BP Neural Network}

Back propagation neural network (BP neural network) is a widely used and researched neural network model, and it is a multilayer feedforward artificial neural network based on the error back-propagation algorithm. The network structure of the typical BP neural network has three or more layers, in which the first layer is input layer and the last layer is the output layer, and the middle layers are the hidden layers (She and Liang, 2007) [11]. This paper studies the BP neural network with three layers, which has only one hidden layer. The input layer contains the 12 indicators of the evaluation of the scientific research projects and has 12 input nodes, 1 output node. In this paper, the BP neural network topology is shown in Figure 2.

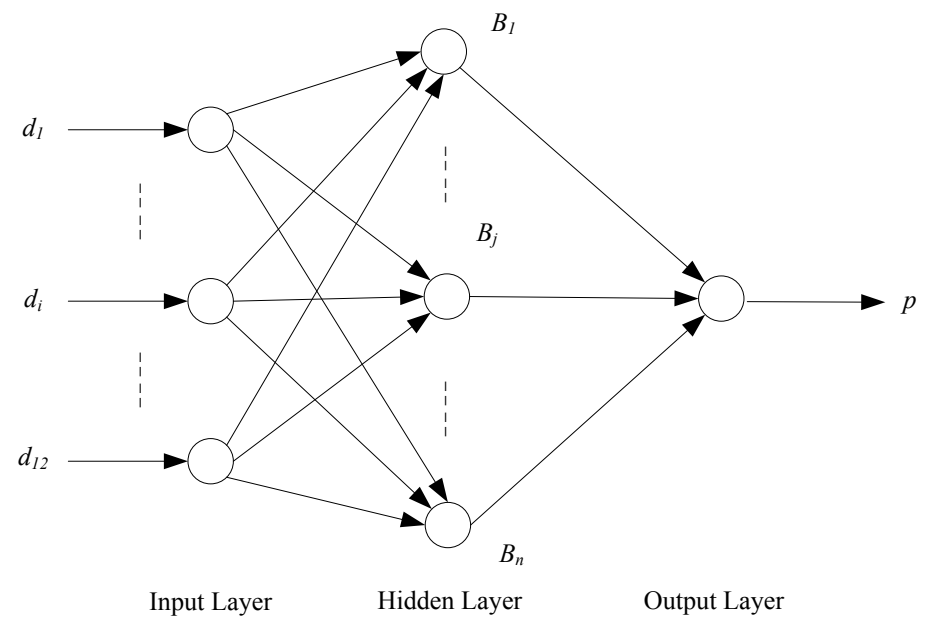

Figure 2. BP neural network topology with three layers

BP neural network calculation process is divided into forward signaling propagation and reverse error propagation processes. In the signaling propagation stage, the input layer needs to address the input information in advance, and then propagate to the hidden layer neurons; the hidden layer is mainly responsible for the information processing and propagates the processed information to the output layer, and output the results after the related process by the output layer. In the error propagation stage, the errors between the actual output and the expected output modify the weights of the input layer and the hidden layer through the output layer in accordance with the error gradient descent. Through modifying the weights of the input layer and the hidden layer, the error in the output layer is gradually reduced and the accuracy of the output result is raised. This process continues to meet the error requirements or the maximum number of calculations.

The output function of the hidden layer in the BP neural network is shown as following: 


$$
B_{j}=f\left(\sum_{i=1}^{t} u_{i j} d_{i}\right)
$$

where $B_{j}$ is the output value of the hidden layer; $u_{i j}$ is connection weights matrix between input layer and hidden layer; $d_{i}$ is the input value; $t$ is the dimension of the input value.

The output function of the BP neural network is shown as following:

$$
p=f\left(\sum_{j=1}^{n} w_{j} B_{j}\right)
$$

where $p$ is the actual output value of the output layer; $f(x)$ is the activation function, normally is sigmoid function, including logarithmic sigmoid function: $f(x)=\frac{1}{1+e^{-x}}$ and the tangent sigmoid function: $f(x)=\frac{1-e^{-x}}{1+e^{-x}} ; w_{j}$ is the connection weight between the hidden layer and the output layer; $n$ is the number of the hidden layers.

The BP neural network built in this paper is based on the Matlab neural network toolbox, and re-program according to the characteristics of the problem to set parameters intelligently. By calculating the scientific research projects automatically, we can select the most suitable hidden layer number and the parameters which need to be determined artificially.

\subsection{Construction of the Linear Neural Network}

The linear neural network is a relatively simple neural network, constituted by one or more linear neurons, the transfer function of each neuron is a linear function, so the output of the network can be arbitrary value. The linear neural network consists of the single-layer linear neural network and multi-layer linear neural network. The network discussed and adopted in this paper is the single-layer linear neural network, and the input node contains 12 indicators, the output node contains 1 indicator. The linear neural network has a wide range of applications, such as in signal filtering, pattern recognition, prediction, and control. The general structure of the single-layer linear neural network is shown in Figure 3. 


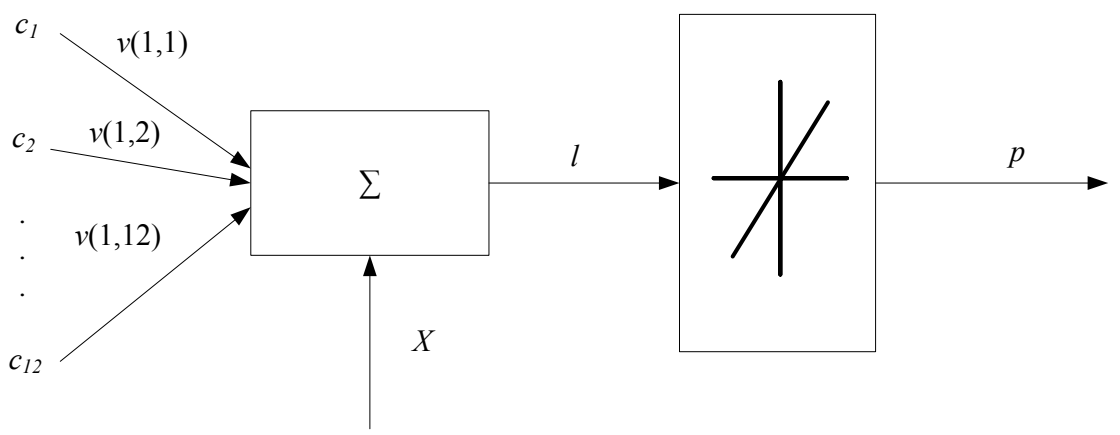

Figure 3. Topology structure of the linear neural network

The output of the linear neural network is:

$$
p=\operatorname{purelin}(v c+X)=v c+X
$$

where purelin(g) is the transfer function of the linear neural network; $v$ is the weight matrix, $c$ is the input vector of the neurons; $X$ is the threshold of the neurons.

The Widrow-Hoff learning rule which is also called LMS (Least Mean Square) can be adopted in the linear neural network to train the weights and the thresholds of one layer and make it linear approximation to a function. The Widrow-Hoff learning rule adopts the gradient descent method, and modified the weights and the thresholds continually along the fastest descent direction of mean squared error (MSE) which makes the output mean squared error minimum. The modifier formulas of the weights and the thresholds in Widrow-Hoff learning rule are shown as following:

$$
\begin{aligned}
& v_{k+1}=v_{k}-\lambda \frac{\partial E_{k}(v, X)}{\partial v}=v_{k}+\lambda\left(Q_{k}-p_{k}\right) c_{k}^{T} \\
& X_{k+1}=X_{k}-\lambda \frac{\partial E_{k}(v, X)}{\partial X}=X_{k}+\lambda\left(Q_{k}-p_{k}\right)
\end{aligned}
$$

where $E_{k}(v, X)$ is the output error function, $Q_{k}$ is the $k$ th expected output, $\lambda$ is the learning rate which determines the convergence speed of the neural network. When $\lambda$ increases, the convergence speed becomes faster, however if $\lambda$ is too large, the learning process will become instability, so we should choose the suitable learning rate according to the practical situation. In the Widrow-Hoff learning rule, the weights and the thresholds of all training samples are modified by Eq. (4) and Eq. (5) and the algorithm stops only when the error accuracy is satisfied or reach the maximum training number.

The linear neural network is constructed in accordance with the above content, and we apply the Matlab neural network toolbox to set the model parameters and obtain the simulation results. 


\subsection{Output Error Function of the Neural Networks}

We adopt the Mean Squared Error (MSE) as the output error function of the BP neural network and linear neural network in this paper, the MSE is shown as following:

$$
m s e=\frac{1}{m} \sum_{k=1}^{m} e^{2}(k)=\frac{1}{m} \sum_{k=1}^{m}\left(Q_{k}-p_{k}\right)^{2}
$$

where mse is the output error of the neural network; $e(k)$ is the error between the $k$ th actual output and expected output; $Q_{k}$ is the $k$ th expected output; $p_{k}$ is the $k$ th actual output; $k=1,2, \ldots, m$, where $m$ is the number of the outputs.

\section{Example Analysis}

After constructing the BP neural network and the linear neural network, we take an evaluation work of the scientific research projects in a university for example. We randomly select 10 projects as the training samples and 5 projects as the testing samples. Training and testing samples are listed in Table 2 and Table 3, the indicators in the tables adopt the 100 scoring method, 100 is the most satisfactory scores, and the value in the table is the average score of five experts.

Table 2. Training samples data

\begin{tabular}{ccccccccccccccc}
\hline \multicolumn{2}{c}{ No. NOT } & FORO & SOTS & DOTI & \multicolumn{2}{c}{ BR } & \multicolumn{2}{c}{ ROER RFOA } & HRST & EC & DOTR DLOAT & IP & Value \\
\hline 1 & 80.9 & 78.6 & 73.2 & 88.7 & 68.9 & 90.2 & 75.5 & 81.1 & 85.6 & 79.9 & 83.5 & 87.7 & 84.6 \\
2 & 75.5 & 79.8 & 83.2 & 84.5 & 86.7 & 72.2 & 75.6 & 88.2 & 73.8 & 69.3 & 80.3 & 77.9 & 80.5 \\
3 & 70.2 & 82.1 & 85.6 & 77.6 & 69.2 & 66.7 & 78.9 & 77.4 & 78.9 & 82.1 & 78.7 & 71.1 & 77.3 \\
4 & 60.1 & 66.2 & 62.1 & 59.5 & 70.2 & 73.5 & 55.6 & 68.9 & 63.6 & 65.8 & 70.3 & 66.9 & 65.2 \\
5 & 83.6 & 80.7 & 77.2 & 60.5 & 75.6 & 68.5 & 72.1 & 77.7 & 75.3 & 70.5 & 80.2 & 75.6 & 73.6 \\
6 & 80.6 & 92.8 & 90.8 & 88.5 & 83.1 & 78.9 & 80.6 & 80.9 & 85.7 & 79.9 & 83.2 & 85.6 & 84.5 \\
7 & 95.3 & 94.5 & 90.2 & 82.6 & 89.6 & 88.8 & 82.5 & 86.4 & 91.2 & 89.9 & 87.5 & 88.6 & 89.3 \\
8 & 83.3 & 77.8 & 75.6 & 78.8 & 80.2 & 83.9 & 88.7 & 78.5 & 77.7 & 75.1 & 78.6 & 80.8 & 79.1 \\
9 & 78.5 & 82.2 & 88.6 & 84.6 & 78.5 & 79.5 & 74.2 & 89.7 & 86.8 & 84.3 & 87.6 & 78.5 & 82.2 \\
10 & 70.1 & 63.2 & 69.8 & 70.5 & 73.6 & 79.8 & 80.5 & 83.2 & 66.6 & 60.2 & 62.5 & 67.5 & 69.3 \\
\hline
\end{tabular}

Table 3. Testing samples data

\begin{tabular}{cccccccccccccc}
\hline \multirow{2}{*}{ No. NOT FORO } & SOTS & \multirow{2}{*}{ DOTI } & \multicolumn{2}{c}{ BR } & \multicolumn{2}{c}{ ROER RFOA } & \multicolumn{2}{c}{ HRST } & EC & DOTRDLOAT & IP & Value \\
\hline 1 & 83.2 & 78.9 & 83.3 & 93.3 & 85.5 & 79.5 & 83.5 & 86.4 & 90.2 & 91.2 & 80.6 & 85.2 & 86.7 \\
2 & 78.5 & 77.8 & 70.1 & 75.6 & 80.3 & 85.6 & 83.2 & 89.4 & 72.1 & 68.8 & 70.1 & 69.7 & 75.8 \\
3 & 72.3 & 60.5 & 66.6 & 76.9 & 77.8 & 69.7 & 62.1 & 56.5 & 60.3 & 72.5 & 63.5 & 72.4 & 66.7 \\
4 & 83.9 & 80.6 & 85.5 & 77.8 & 76.9 & 83.3 & 69.9 & 76.8 & 85.7 & 86.9 & 84.2 & 83.6 & 81.2 \\
5 & 73.5 & 69.7 & 82.6 & 81.4 & 74.9 & 75.6 & 79.3 & 80.2 & 86.9 & 78.6 & 67.8 & 70.1 & 76.8 \\
\hline
\end{tabular}


We input the above training and testing samples data to the prepared Matlab program, and according to the construction idea of the neural network models shown in Figure 1, we obtain the computed results of two kinds of neural networks which are showed in Table 4. In order to compare with the results of the neural networks, we also provide the result of linear regression forecasting in Table 4.

Table 4. Computed result of testing samples

\begin{tabular}{cccccc}
\hline & \multicolumn{5}{c}{ No. } \\
\cline { 2 - 6 } & 1 & 2 & 3 & 4 & 5 \\
\hline Expected value & 86.7 & 75.8 & 66.7 & 81.2 & 76.8 \\
BP neural network & 88.6 & 75.4 & 65.7 & 76.5 & 74.3 \\
Linear neural network & 89.5 & 76.7 & 64.3 & 83.4 & 69.3 \\
Linear regression & 96.1 & 74.5 & 74.6 & 82.2 & 78.4 \\
$\quad$ forecasting & & & & \\
\hline
\end{tabular}

We adopt the Relative error (RE) and mean absolute percentage error (MAPE) index to show the accuracy of the three methods. The RE and MAPE are shown in Table 5.

Table 5. Relative error and MAPE of outputs in different methods

\begin{tabular}{ccccccc}
\hline \multirow{2}{*}{ Relative Error } & \multicolumn{7}{c}{ No. } \\
\cline { 2 - 7 } & 1 & 2 & 3 & 4 & 5 & MAPE \\
\hline BP neural network & $-2.19 \%$ & $0.53 \%$ & $1.50 \%$ & $5.79 \%$ & $3.26 \%$ & $2.65 \%$ \\
Linear neural network & $-3.23 \%$ & $-1.19 \%$ & $3.60 \%$ & $-2.71 \%$ & $9.77 \%$ & $4.10 \%$ \\
Linear regression \\
$\quad$ forecasting & $-10.84 \%$ & $1.72 \%$ & $-11.84 \%$ & $-1.23 \%$ & $-2.08 \%$ & $5.54 \%$ \\
\hline
\end{tabular}

We can see from the computed results in Table 4 and Table 5 that BP neural network, linear neural network and linear regression forecasting could get the relatively good results. The relative error in BP neural network is relatively stable while the No. 5 testing sample in linear neural network and the No.1 and No.3 testing samples in linear regression forecasting are much higher than the other values. The MAPE of BP neural network is lower than the other two methods and the MAPE of linear regression forecasting is the highest. It shows that both of the neural network methods could get better prediction results than the linear regression forecasting even with this small example. The reason is that the relationship between the indexes and the evaluation results may not be simple linear relation but nonlinear relation. Because of the un-known nonlinear relation between the indexes and the evaluation results, the evaluation results even are not true when we specify a nonlinear construction by using the traditional nonlinear regression prediction method. We can also deduce that if the scale of the example is bigger, the advantages of the neural network will be more obvious.

We can see compare the outputs of BP neural network, linear neural network, linear regression forecasting and the expected values clearly and visually in Figure 4. 


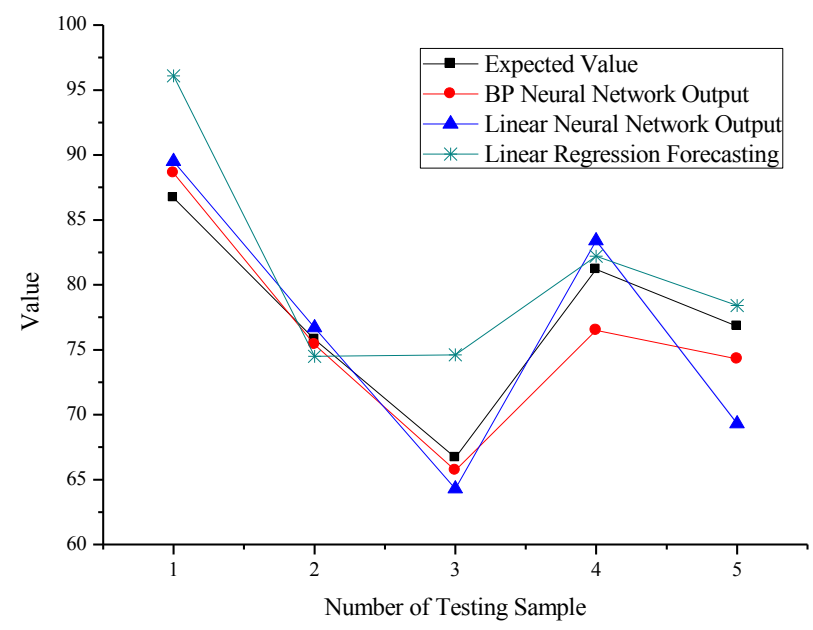

Figure 4. Relationship between the outputs and expected values

From Figure 4, we can see the tendency of the expected values in different testing samples. By comparing the curves of the outputs of the BP neural network, the linear neural network, linear regression forecasting and the expected values, we can see that the tendency of BP neural network is much more similar to the tendency of the expected values than the tendency of the linear neural network and linear regression forecasting. This also shows that the outputs of the BP neural network have higher accuracy than the linear neural network and linear regression forecasting.

The performances of the BP neural network and the linear network are showed in Figure 5 and Figure 6.

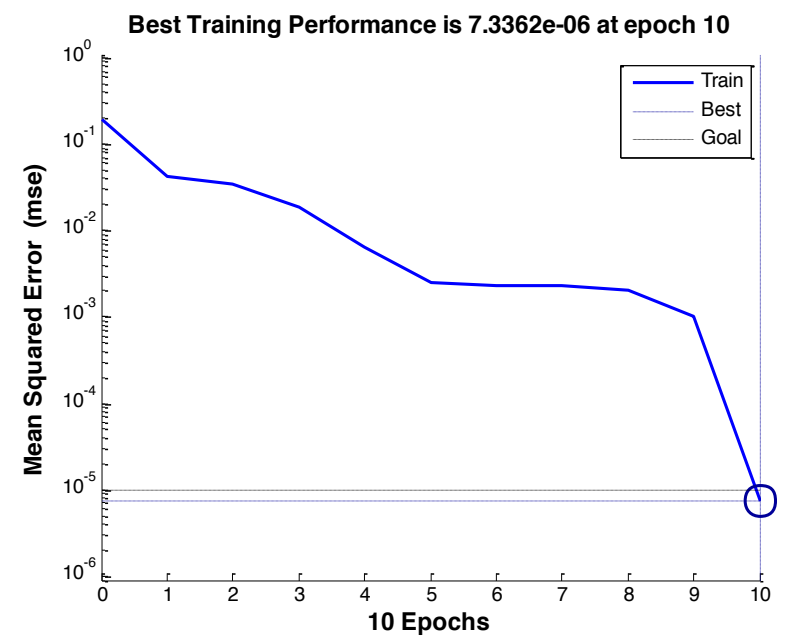

Figure 5. Computed result of the BP neural network 


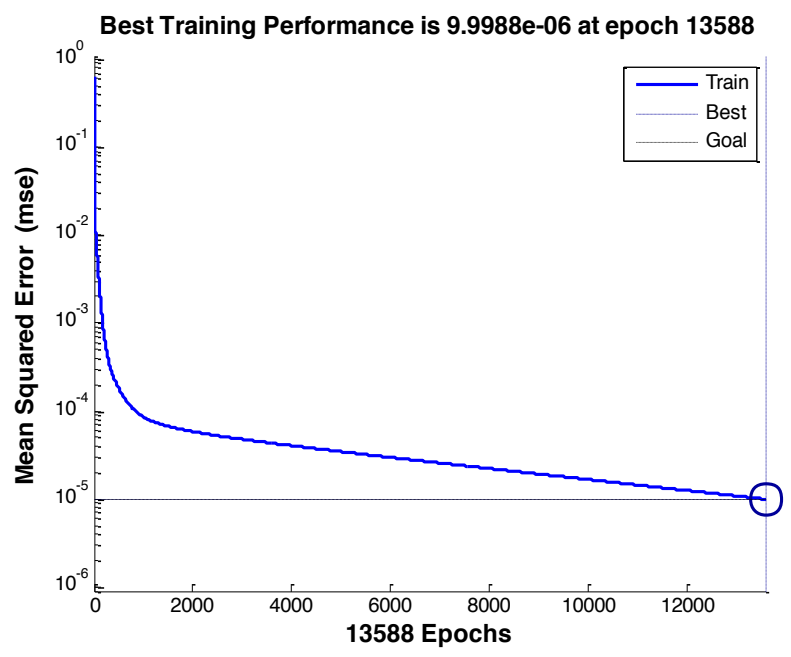

Figure 6. Computed result of the linear neural network

It can be seen from the computed results of two built neural networks in Figure 5 and Figure 6, the linear neural network needs 13588 epochs to achieve the accuracy of $10^{-5}$, while the BP neural network only need 10 epochs to achieve the accuracy of $10^{-5}$. Therefore, we can see that, for the evaluation of the scientific research projects problem, the BP neural network could meet the accuracy requirements within a short period of time and less number of operations, we choose the BP neural network as the optimal network. For the other scientific research projects to be evaluated in the future, based on the established BP neural network in this paper, we can get the final computed results of the evaluation of the scientific research projects by using simulation calculation.

\section{Conclusion}

The evaluation of the scientific research projects is necessary and important in optimizing the allocation of the scientific research resources. The recent research findings are analyzed and the comprehensive evaluation index system of the scientific research projects which includes 12 indexes is built. The research ideas are designed according to the features of the evaluation of the scientific research projects, the basic principle of the neural network is introduced, and the BP neural network model and the linear neural network model are set up, and the output error function of the proposed neural networks is introduced. Finally, by using the Matlab software, two built neural networks are trained with 10 training samples and 5 testing samples of the scientific research projects. Through the comparative analysis of the two proposed neural network and linear regression forecasting, the BP neural network is selected as the optimal network, and used by the evaluation of the scientific research projects in the future. From the simulation results, we can see that the BP neural network has higher speed of calculation and accuracy, and could meet the requirements of the evaluation of the scientific research projects, and could provide some assistance for the decision-making of universities, research institutions and other related departments. 
From this study, we can get some advantages by using the neural network methods to evaluate the scientific research projects: (1) It only needs part of the projects to get the relationship between the complex indexes and the evaluation result, and evaluate the remaining projects by using the trained neural networks, while it needs to get all the index values of all the projects in the traditional methods; (2) It is not necessary to specify the function (eg. linear or nonlinear function) or relationship for the inputs and the outputs, and the relationship in the neural networks are kept in the weights of hidden layers; (3) It is maybe useful to evaluate the projects which have a large-scale of applicants and proposals and save time and money costs in the evaluation process.

\section{Acknowledgments}

It is supported by the Key Project of Natural Science Foundation of China (71132008) and the Fundamental Research Funds for the Central Universities (2011JBM173).

\section{References}

[1] Cao Z., Chai C., Application of fuzzy multilevel comprehensive evaluation method in assessment of scientific research subjects, Aeronautical Computer Technique, 37, 1, 2007, 51-53. (In Chinese)

[2] Carlsson C., Fullér R., Majlender P., A fuzzy approach to R\&D project selection, EUSFLAT Conference, 2005: 536-540.

[3] Henriksen A.D., Traynor A. J., A practical R\&D project-selection scoring tool, IEEE Transactions on Engineering Management, 46, 2, 1999: 158-170.

[4] Liberatore M. J., An extension of the analytic hierarchy process for industrial R\&D project selection and resource allocation, IEEE Transactions on Engineering Management, 34, 1, 1987: 12-18.

[5] Lin H., Study on evaluation of scientific and technical projects with set pair analysis research, Science Technology and Engineering, 5, 19, 2005, 1365-1368. (In Chinese)

[6] Lin H., Scientific research projects evaluation and selection based on the TOPSIS method, Science \& Technology Progress and Policy, 23, 7, 2006, 47-49. (In Chinese)

[7] Meade L. M., Presley A., R\&D project selection using the analytic network process, IEEE Transactions on Engineering Management, 49, 1, 2002: 59-66.

[8] Mohanty R. P., Agarwal R., Choudhury A. K., Tiwari M.K., A fuzzy ANP-based approach to R\&D project selection: a case study, International Journal of Production Research, 43, 24, 2005: 5199-5216.

[9] Pan J., Liu X., Study on evaluation index system and the fuzzy optimization model of science research projects, Science of Science and Management of S.\& T., 25, 1, 2004, 911. (In Chinese)

[10] Schmidt R. L., Freeland J. R., Recent progress in modeling R\&D project-selection processes, IEEE Transactions on Engineering Management, 39, 2, 1992: 189-201.

[11] She C., Liang X., Evaluation on the scientific research project based on ENN, Forum on Science and Technology in China, 6, 2007, 107-109. (In Chinese) 
[12] Song Z., Wang Y., Scientific research projects evaluation based on weighted optimum order numbers, Electronic Design Engineering, 19, 24, 2011, 66-68. (In Chinese)

[13] Wang Y., Song Z., Evaluation method of scientific research projects based on the FAHP method, Market Modernization, 21, 2008, 25-26. (In Chinese)

This paper is a revised and extended version of work originally presented at the 2013 International Conference on Logistics, Informatics and Services Sciences (LISS 2013) 21-24 August 2013, Reading, UK 